\title{
Organizaciones intensivas en conocimiento (oIC): características e implicaciones para la gestión
}

\author{
Cristian Bedoya Dorado* \\ Mónica García Solarte** \\ Guillermo Murillo Vargas***
}

Fecha de recibido: 13 de febrero de 2020 Fecha de aprobado: 6 de octubre de 2021

Para citar este artículo: Bedoya Dorado, C., García Solarte, M., \& Murillo Vargas, G. (2021). Organizaciones intensivas en conocimiento (oIC): características e implicaciones para la gestión. Revista Universidad \& Empresa, 23(41), 1-34. https//doi.org/10.12804/

revistas.urosario.edu.co/empresa/a.8701

\section{Resumen}

A finales del siglo xx fue anunciada la llegada de un nuevo tipo de organización que estaría fundamentada en el conocimiento como núcleo de su estrategia. En la literatura, este tipo de organización se ha registrado principalmente como organizaciones intensivas en conocimiento (orc). Pese al papel que cumplen las

* Grupo Humanismo y Gestión, Departamento de Administración y Organizaciones, Facultad de Ciencias de la Administración, Universidad del Valle. Correo electrónico: bedoya.cristian@correounivalle.edu.co ORCID: http://orcid. org/0000-0001-9609-0319

** Grupo Humanismo y Gestión, Departamento de Administración y Organizaciones, Facultad de Ciencias de la Administración, Universidad del Valle. Correo electrónico: monica.garcia@correounivalle.edu.co ORCID: https://orcid. org/0000-0001-6219-0012

*** Grupo Humanismo y Gestión, Departamento de Administración y Organizaciones, Facultad de Ciencias de la Administración, Universidad del Valle. Correo electrónico: guillermo.murillo@correounivalle.edu.co ORCID: https://orcid. org/0000-0003-2268-598X 
OIc en el actual contexto, su definición ha resultado ser difusa y variable en la literatura. El presente estudio se enfoca en identificar las características de las orc y sus implicaciones para la gestión. Para ello, el estudio se abordó desde un diseño documental en el que se realizó una revisión sistemática de la literatura mediante un análisis de contenido cuantitativo/cualitativo de una muestra de 509 artículos de los catálogos de Scopus y Web of Science. Los hallazgos sugieren que las confusiones teóricas se deben en gran medida a que la literatura registra diferentes etiquetas para conceptualizar e investigar a las orc. El conocimiento es una de las principales características, ya que es la fuente de ventaja competitiva, y sitúa los tipos de trabajadores, los productos y el modo de organizar a estas organizaciones. Las características de las orc las diferencian de organizaciones tradicionales, lo que sugiere nuevas formas de gestión.

Palabras clave: organizaciones intensivas en conocimiento; conocimiento; economía del conocimiento; innovación.

\title{
Knowledge-Intensive Organizations (KIOS): Characteristics and Implications for Management
}

\begin{abstract}
At the end of the $20^{\text {th }}$ century, the arrival of a new type of organization was announced, which would be based on knowledge as the core of its strategy. In the literature this type of organization has been registered mainly as knowledge-intensive organizations (kios). Despite the role played by kios in the current context, their definition has proven to be diffuse and variable in the literature. This study focuses on identifying the characteristics of kios and their implications for management. For this, the study was approached from a documentary design in which a systematic review of the literature was carried out through a quantitative/qualitative content analysis of a sample of 509 articles from the Scopus and Web of Science catalogs. The findings suggest that the theoretical confusions are largely since the literature registers different labels for conceptualizing and investigating KIOs. Knowledge is one of the main characteristics, since it is the source of competitive advantage, and it locates the types of workers, the products, and the way of organizing these organizations. The characteristics of kIos differentiate them from traditional organizations, suggesting new forms of management.
\end{abstract}

Keywords: Knowledge-intensive organizations; knowledge; knowledge economy; innovation.

\section{Organizações intensivas de conhecimento (oIc): características e implicações para a gestão}

\section{Resumo}

No final do século 20, foi anunciada a chegada de um novo tipo de organização, que teria o conhecimento como cerne de sua estratégia. Na literatura, este tipo de organização tem sido registrada principalmente como organizações intensivas de conhecimento (oIc). Apesar do papel desempenhado pelas orc no contexto atual, sua definição tem se mostrado difusa e variável na literatura. Este estudo enfoca a identificação das características das orc e suas implicações para a gestão. Para tanto, o estudo foi abordado a partir de um desenho documental em que foi realizada uma revisão sistemática da literatura por meio de uma análise de conteúdo quantitativa/ qualitativa de uma amostra de 509 artigos dos catálogos Scopus e Web of Science. Os resultados sugerem que as confusões teóricas são em grande parte devido ao fato de que a literatura registra diferentes rótulos para conceituar e investigar as orc. O conhecimento é uma das principais características, pois é fonte de vantagem competitiva, e localiza os tipos de trabalhadores, os produtos e a forma de organização dessas organizações. As características das oIc as diferenciam das organizações tradicionais, sugerindo novas formas de gestão.

Palavras-chave: organizações intensivas em conhecimento; conhecimento; economia do conhecimento; inovação. 


\section{Introducción}

A partir de los años noventa del siglo pasado, la economía mundial se ha caracterizado, entre diferentes razones, por el constante crecimiento del sector servicios, sus actividades comerciales y el aumento de la generación de empleos. En este sector se han concentrado compañías e industrias dedicadas a explotación del conocimiento y el uso intensivo de las tecnologías (Banco Mundial, 2019). Dicho fenómeno ha sido abordado principalmente desde la noción de sociedad del conocimiento, bajo la cual la literatura académica ha registrado un tipo de organización diferente a las organizaciones de los sectores industriales, cuya fuente de riqueza es el conocimiento (Grey \& Sturdy, 2009; Makani \& Marche, 2010; Naisbitt, 1983; Sveiby, 2001; Wyrwich, 2019). De acuerdo con Greenwood (2009), la sociedad del conocimiento como sociedad posindustrial es más intensiva en conocimiento que la sociedad industrial, la cual ha dado origen a organizaciones intensivas en conocimiento (oIc).

En este tipo de organizaciones, los activos intangibles como el conocimiento, los procesos de know-how, las mejores prácticas (best practices) y el capital intelectual adquieren mayor relevancia a diferencia de las organizaciones tradicionales de sociedad industrial (Drucker, 2002; Krüger, 2006; Lundvall \& Borrás, 1997; Makani \& Marche, 2010). Es por ello que, para Lundvall (1999), las orc son organizaciones que tienen como objeto y recurso principal la transformación del conocimiento para obtener innovación, aprendizaje y mejora de técnicas, que son aplicados para producir nuevo conocimiento en proyectos de innovación, investigación y desarrollo (I+D), y de formación y aprendizaje.

Este tipo de organizaciones se empiezan a registrar en la literatura académica a finales del siglo $\mathrm{xx}$, destacando que el conocimiento es el elemento diferenciador y su gestión es el núcleo de la estrategia organizacional (Makani \& Marche, 2010; Sveiby, 2001). Diversos autores coinciden en que William H. Starbuck es el primer autor en introducir el concepto OIC en el contexto norteamericano (Alvesson, 1993, 2000, 2001; Blackler, Reed \& Whitaker, 1993; Ditillo, 2004; Makani \& Marche, 2010; Robertson \& Swan, 2003; Swart \& Kinnie, 2003). Sin embargo, Starbuck (1992) en su primera publicación define las orc a partir de discusiones que años anteriores había planteado y publicado Karl E. Sveiby sobre las industrias intensivas en conocimiento o knowledge-intensive industries en el contexto académico sueco, tanto en su lengua materna como en inglés (Sveiby, 1992; Sveiby \& Lloyd, 1987). Como 
consecuencia, de las bases teóricas propuestas por Starbuck, que incluían las de Sveiby, derivan diversos términos para comprender las oIc, como knowledge-intensive organization (кIO), knowledge-intensive company (KIC), knowledge-intensive firms (KIF), knowledge-based organization (кво), knowledge-intensive business (кІв) (Alvesson, 1993, 2011; Deng, 2008; Kuivalainen, Sundqvist, Puumalainen \& Cadogan, 2004; Kvaløy \& Olsen, 2008).

La variedad de etiquetas o términos para estudiar las oic ha generado ciertas controversias teóricas y conceptos vagos (Alvesson, 2011; Blackler, 1995; Grey \& Sturdy, 2009; Kemp, Moerman \& Prieto, 2001; Makani \& Marche, 2010, 2012; Song, Zhang \& Shi, 2007; Starbuck, 1992). En la investigación de Makani y Marche (2010), se encuentra que existe una falta de consenso entre los académicos y profesionales sobre la definición de oic. Según ellos, pese a que es posible establecer diferencias entre una oIc y una organización tradicional, surgen discrepancias a la hora de caracterizarlas. Uno de los argumentos que señalan los autores es la falta de claridad que hay acerca de las actividades que efectúan los trabajadores de las OIC y de las organizaciones tradicionales (Makani \& Marche, 2010).

En la revisión de la literatura de Makani y Marche (2010), se concluye que las oic son un tipo de organización en la que el conocimiento es tanto una entrada como una salida y, por consiguiente, un recurso vital. Autores como Alvesson (1993) sostienen que la noción de conocimiento y su uso intensivo son la base para identificar un grupo de trabajadores (denominados como trabajadores del conocimiento) o un tipo de organización que permite su diferenciación de otro tipo de trabajadores y organizaciones. Sin embargo, es difícil definir el conocimiento de una manera abstracta y no radical. Para los años noventa, autores como Winter (1998) identificaban una insuficiencia del lenguaje y una carencia de terminología y de los esquemas conceptuales apropiados a la hora de analizar el papel que desempeña el conocimiento en la economía. Por su parte, en los 2000, Greenwood (2009) argumentaba que tanto la literatura de la sociedad del conocimiento como la de las OIC se encontraban en un terreno intelectual caótico, debido a que diferentes disciplinas y actores no académicos habían participado en múltiples dimensiones de su estudio.

El actual contexto del mundo de los negocios y las economías, situado en una Cuarta Revolución Industrial, que se caracteriza por la automatización de los procesos productivos, la realidad virtual, la inteligencia artificial, el aprendizaje de las máquinas, el avance tecnológico, la disponibilidad de los datos, la configuración de las prácticas de gestión del 
conocimiento, etc. (Castellani et al., 2020; Deloitte Insights, 2019), requiere de discusiones conceptuales que permitan comprender cuáles son las características de los nuevos modelos de organizaciones, al tiempo que permita contribuir a la literatura académica, la cual desde los años noventa ha venido abordando el modo en que el conocimiento configura un tipo de organizaciones que hace uso intensivo de él. Teniendo en cuenta lo anterior, el objetivo del presente texto es identificar las características de las oIC y sus implicaciones para la gestión. El ejercicio investigativo se sustenta en un diseño documental bajo el método de la revisión sistemática de la literatura, en la cual se llevó a cabo un análisis de contenido cuantitativo y cualitativo. El material de análisis consiste en artículos publicados en los catálogos digitales de Scopus y Web of Science. Las características de las orc se establecieron mediante categorías emergentes de los artículos y sus frecuencias permitieron identificar tendencias en las discusiones de los autores.

La estructura de este artículo se divide en cuatro partes: primero, se exponen los aspectos metodológicos y la construcción del material bibliográfico para la revisión de la literatura. Seguido, son presentados los resultados del análisis de contenido en las definiciones encontradas. Tercero, los resultados de las características de las oic son discutidos en comparación con las organizaciones tradicionales. Finalmente, se señalan las conclusiones, limitaciones e implicaciones de la investigación para el campo de la administración y las organizaciones, y se ofrecen recomendaciones para futuras líneas de investigación.

\section{Metodología}

La investigación se abordó desde un diseño documental, en el que se empleó como técnica de recolección de la información la selección de material bibliográfico, que, según Mayan (2009), es una de las estrategias más comunes para la obtención de datos. Este tipo de información permite conocer el contexto de investigación, así como rastrear el desarrollo de un tema a partir de múltiples fuentes, entre las que se encuentran las noticias, reportes, libros, artículos, piezas históricas, etc. El enfoque de investigación fue cualitativo y el tipo de investigación descriptivo-analítico. De acuerdo con Méndez (2006), el estudio 
descriptivo permite identificar características asociadas a los elementos y componentes del universo de investigación.

El método utilizado fue la revisión sistemática de la literatura, que, conforme con Fink (2005), se caracteriza por ser no solo sistemático, sino también explícito y reproducible para la identificación, evaluación y agrupación de un corpus existente de material bibliográfico sobre un fenómeno de investigación. Por su parte, Meredith (1993) considera que este tipo de métodos posibilita reprocesar el estado actual de las investigaciones acerca de un tema particular, ya que ofrece un resumen de los tópicos y perspectivas de dicho tema, y permite identificar el contenido teórico y metodológico de estos. La revisión sistemática de la literatura se desarrolló siguiendo tres fases: búsqueda y selección del material, construcción de una plantilla de organización del material y de las categorías de análisis, y análisis de la información.

\subsection{Búsqueda y selección del material}

Para la recolección de datos se emplearon los catálogos de Scopus y Web of Science, los cuales agrupan las principales colecciones de datos de referencias bibliográficas y de citas, que poseen métricas para evaluar el impacto y la calidad de las referencias. Por una parte, Scopus es una base de datos de la empresa Elsevier que cuenta con referencias con revisión por pares (peer review) y contenido de calidad, así como la métrica de impacto scimago Journal Rank (SJR). Por otra parte, Web of Science, propiedad de Clarivate Analytics, cuenta con el Journal Citation Reports y los Essential Science Indicators. Ello permite realizar la revisión de la literatura con publicaciones de calidad e impacto en el contexto de la academia y la investigación.

En dichos catálogos se seleccionaron artículos que tuvieran en sus títulos algunas de las etiquetas de las oIc: 'knowledge-intensive organizations', 'knowledge-intensive firms', 'knowledge companies', 'knowledge-based organizations' y 'knowledge-intensive business'. Para elegir las publicaciones se tuvieron en cuenta los siguientes criterios:

- Inclusión: a) artículos (empíricos, de revisión o de reflexión); b) publicados en inglés o español; c) publicaciones que discutieran sobre las oIc sin importar el tipo de términos o etiqueta; d) publicaciones desde 1992, año 
en el que aparecen los trabajos fundacionales de Sveiby (1992) y Starbuck (1992), hasta 2020, el último año transcurrido para comparar el total de publicaciones en dicho período.

- Exclusión: a) documentos procedentes de eventos académicos, libros, capítulos de libros, reseñas de libros, etc.; b) artículos publicados en idiomas diferentes al inglés o español; c) publicaciones que no tuvieran a las oIC como unidad de análisis.

Las publicaciones encontradas fueron organizadas en una base de datos, y se excluyeron las duplicidades debido a que algunos artículos estaban indexados en los dos catálogos digitales. Como resultado se obtuvo un total de 509 artículos académicos que cumplían con los criterios de inclusión y exclusión. En la figura 1 se presenta el total de publicaciones por año.

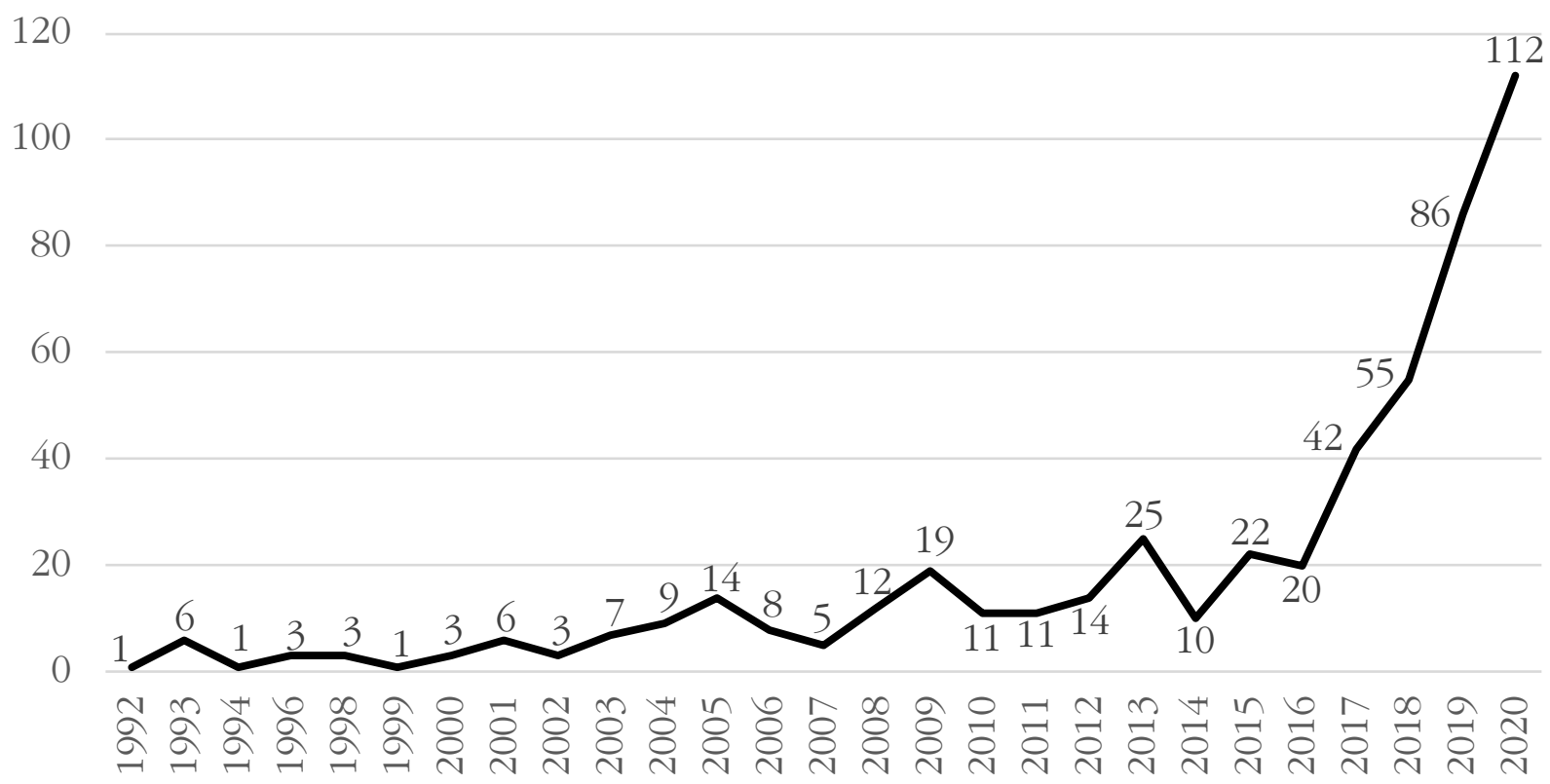

Figura 1. Publicaciones por año (1992-2020)

Fuente: elaboración de los autores. 


\subsection{Plantilla de organización y construcción de categorías}

A partir de las publicaciones seleccionadas se construyó una plantilla para organizar la información de estas. Se definieron como variables descriptivas el término o la etiqueta utilizados en las publicaciones para mencionar a las oIc, la región geográfica a la cual pertenece, la afiliación institucional del autor o de los autores de cada publicación, y el tipo de estudio según su enfoque. Así mismo, se establecieron seis categorías de análisis: competencia y estrategia, conocimiento, contexto, estructura, procesos y productos, $y$ trabajadores, con el objetivo de caracterizar a las oic. Estas características se plantearon de acuerdo con la revisión en la literatura elaborada por Greenwood (2009), Kemp et al. (2001) y Makani y Marche $(2010,2012)$ (ver tabla 1).

Tabla 1. Sistematización de las publicaciones seleccionadas

\begin{tabular}{|c|c|c|c|c|}
\hline Año de publicación & $\begin{array}{l}\text { 1992-2004 } \\
\text { (12 años) } \\
\text { Período de } \\
\text { incubación } \\
\mathrm{N}=43(8 \%)\end{array}$ & $\begin{array}{c}2005-2011 \\
\text { (6 años) } \\
\text { Período de } \\
\text { crecimiento } \\
\mathrm{N}=80(16 \%)\end{array}$ & $\begin{array}{c}2012-2020 \\
\text { (8 años) } \\
\text { Período de } \\
\text { dominio } \\
\mathrm{N}=386(76 \%)\end{array}$ & $\begin{array}{c}1992-2020 \\
(28 \text { años }) \\
\text { Total } \\
N=509(100 \%)\end{array}$ \\
\hline \multicolumn{5}{|l|}{ Etiqueta } \\
\hline Knowledge-based business & $1(2.3)$ & $0(0)$ & $16(4.1)$ & $17(3.3)$ \\
\hline Knowledge-based firms & $7(16.3)$ & $0(0)$ & $4(1)$ & $11(2.2)$ \\
\hline Knowledge-based organization & $5(11.6)$ & $6(7.5)$ & $3(0.8)$ & $14(2.8)$ \\
\hline Knowledge-intensive business & $1(2.3)$ & $0(0)$ & $41(10.6)$ & $42(8.3)$ \\
\hline Knowledge-intensive business service & $4(9.3)$ & $36(45)$ & $127(32.9)$ & $167(32.8)$ \\
\hline Knowledge-intensive companies & $0(0)$ & $2(2.5)$ & $16(4.1)$ & $18(3.5)$ \\
\hline Knowledge-intensive firm & $20(46.5)$ & 25 (31.3) & $70(18.1)$ & $115(22.6)$ \\
\hline knowledge-intensive industries & $0(0)$ & $0(0)$ & $3(0.8)$ & $3(0.6)$ \\
\hline Knowledge-intensive organizations & $5(11.6)$ & $9(11.3)$ & $93(24.1)$ & $107(21)$ \\
\hline Knowledge-intensive SMES & $0(0)$ & $2(2.5)$ & $13(3.4)$ & $15(2.9)$ \\
\hline \multicolumn{5}{|l|}{ Región geográfica } \\
\hline África & $0(0)$ & $1(1.3)$ & $1(0.3)$ & $2(0.4)$ \\
\hline Asia & $3(7)$ & $9(11.3)$ & $31(8)$ & $43(8.4)$ \\
\hline Europa & $29(67.4)$ & 41 (51.3) & $217(56.2)$ & $287(56.4)$ \\
\hline Norteamérica & $8(18.6)$ & $28(35)$ & $106(27.5)$ & $142(27.9)$ \\
\hline Suramérica & $0(0)$ & $0(0)$ & $12(3.1)$ & $12(2.4)$ \\
\hline Oceanía & $3(7)$ & $1(1.3)$ & $19(4.9)$ & $23(4.5)$ \\
\hline \multicolumn{5}{|l|}{ Tipos de estudio } \\
\hline Cualitativo & 35 (81.4) & 47 (58.8) & $215(55.7)$ & $297(58.3)$ \\
\hline Cuantitativo & $8(18.6)$ & $33(41.3)$ & $158(40.9)$ & $199(39.1)$ \\
\hline Mixto & $0(0)$ & $0(0)$ & $13(3.4)$ & $13(2.6)$ \\
\hline
\end{tabular}




\begin{tabular}{|c|c|c|c|c|}
\hline Año de publicación & $\begin{array}{c}1992-2004 \\
\text { (12 años) } \\
\text { Período de } \\
\text { incubación } \\
\mathrm{N}=43(8 \%)\end{array}$ & $\begin{array}{c}2005-2011 \\
\text { (6 años) } \\
\text { Período de } \\
\text { crecimiento } \\
\mathrm{N}=80(16 \%)\end{array}$ & $\begin{array}{c}2012-2020 \\
\text { (8 años) } \\
\text { Período de } \\
\text { dominio } \\
\mathrm{N}=386(76 \%)\end{array}$ & $\begin{array}{c}1992-2020 \\
(28 \text { años }) \\
\text { Total } \\
N=509(100 \%)\end{array}$ \\
\hline \multicolumn{5}{|l|}{ Característica oIC } \\
\hline Competencia y estrategia & $5(11.6)$ & $17(14.2)$ & $86(16.7)$ & $108(15.7)$ \\
\hline Conocimiento & $23(53.5)$ & $32(26.7)$ & $226(43.9)$ & $281(40.8)$ \\
\hline Contexto & $1(2.3)$ & $16(13.3)$ & $59(11.5)$ & $76(11)$ \\
\hline Estructura & $13(30.2)$ & $22(18.3)$ & $32(6.2)$ & $67(9.7)$ \\
\hline Procesos y productos & $6(14)$ & $19(15.8)$ & 75 (14.6) & $100(14.5)$ \\
\hline Trabajadores & $5(11.6)$ & $14(11.7)$ & $37(7.2)$ & $56(8.1)$ \\
\hline
\end{tabular}

Fuente: elaboración de los autores.

Con el objetivo de identificar las frecuencias y tendencias de las características de las publicaciones sobre orc y de este tipo de organizaciones, se efectuó un análisis de contenido. De acuerdo con Aktouf (2011), este tipo de análisis busca identificar cantidades, enumerarlas y definir frecuencias de la aparición de elementos definidos como unidades de información o de significación. Según este autor, lo más significativo es aquello que aparece con mayor frecuencia. Para llevar a cabo este ejercicio, se desarrolló una codificación en la que los conceptos y argumentos planteados por los autores de las publicaciones se agruparon conforme con sus propiedades y dimensiones en las categorías establecidas. De este modo, cada identificación y agrupación de las publicaciones permitió contabilizar las frecuencias de cada categoría.

En la plantilla, las variables y categorías se agruparon por períodos de años para identificar el comportamiento de las publicaciones, y se presentan en frecuencias y en porcentajes (ver tabla 1). La organización de las publicaciones permite identificar que de 2012 a 2020 se encuentra el mayor crecimiento en el número de los artículos sobre OIC (76\%), el cual contrasta con los doce primeros años de las publicaciones, que abarcan tan solo el 8\% del total de artículos. Aproximadamente el 56\% del total de publicaciones son realizadas por autores afiliados en el contexto europeo y el $28 \%$ por autores afiliados en Norteamérica. Las publicaciones se caracterizan por ser principalmente estudios de enfoques cualitativos (58.3\%). 


\section{Análisis de la información}

Una vez determinadas las frecuencias de las categorías de análisis, se hizo un análisis de contenido cualitativo. Este tipo de análisis se orienta a los temas, palabras o conceptos presentes en el material seleccionado. A diferencia del análisis de contenido cuantitativo, se busca más que las frecuencias o porcentajes, la novedad, los significados y todos los elementos semánticos relacionados con el material de análisis (Aktouf, 2011). Para este proceso se revisó la información codificada, de modo que se pudieran describir y analizar las características que en las publicaciones se mencionan de las orc. Para ello, se plantearon preguntas orientadoras como: ¿cuáles son las estrategias que emplean las oic para com-

petir?, ¿cuáles son las características del conocimiento para la literatura sobre las oIc y de qué manera este es gestionado en ellas?, ¿cuál es el contexto en el cual se circunscriben las orc?, ¿cuáles son los procesos y tipo de productos que desarrollan las oic? y ¿qué papel juegan los trabajadores y cómo son definidos para este tipo de organizaciones?

Los resultados son presentados y discutidos de acuerdo con las etiquetas o términos registrados en la literatura académica seleccionada, y las seis categorías establecidas en la tabla 1 (características de las orc). Las frecuencias de cada una de estas permitieron orientar la discusión sobre los elementos que los autores del campo de las oic han resaltado y posicionado como los más significativos.

\section{Resultados}

\subsection{Etiquetas y tipos de oIC}

La noción de oic se encuentra en la literatura referenciada a partir de diferentes términos. Como se describe en la tabla 1, las etiquetas más frecuentes son las de knowledge-intensive business service (KIBS) (32.8\%) y knowledge-intensive firm (22.6\%). La literatura no especifica cuáles son las diferencias entre estos términos, sino que son empleados como sinónimos. Por ejemplo, Alvesson (1993, 1995, 2000, 2001, 2011), quien ha sido uno de los autores con mayor número de publicaciones sobre las oic, en sus primeros trabajos 
emplea el término KIF y en los últimos, el término KIO. Estos cambios se pueden observar también según los tres períodos de las publicaciones de la tabla 1, en la que el período de incubación el término con mayor frecuencia en las publicaciones es el de KIF (46.5\%); en el período de crecimiento incremental, el de KIBS (45\%); y en el período de crecimiento sustancial, el de кIBS (32.9\%) у кіо (24.1\%). En la revisión realizada por Makani y Marche (2010), también es posible identificar el empleo de dichos términos como sinónimos, cuando establecen los factores que definen las oic.

Lo anterior respalda los argumentos ya mencionados en los que diversos autores reconocen el problema de la conceptualización de las orc tanto por las distintas formas de abordarlas como por los distintos actores que intervienen en su aproximación (Greenwood, 2009; Makani \& Marche, 2010; Winter, 1998). En la tabla 2 se muestran algunas características de las etiquetas exploradas en la revisión de la literatura.

Tabla 2. Etiquetas de las olc

\begin{tabular}{|c|c|}
\hline Etiqueta o término & Referencia \\
\hline \multicolumn{2}{|l|}{ Knowledge-based business } \\
\hline $\begin{array}{l}\text { Negocios que emplean la gestión del conocimiento } \\
\text { en el contexto de una economía del conocimiento en } \\
\text { desarrollo. }\end{array}$ & $\begin{array}{l}\text { (Herstad \& Ebersberger, 2015; Horváth \& Berbegal- } \\
\text { Mirabent, 2020; Shi, Wu \& Zhao, 2014). }\end{array}$ \\
\hline \multicolumn{2}{|l|}{ Knowledge-based firms } \\
\hline $\begin{array}{l}\text { Organizaciones que transforman sus estructuras de mo- } \\
\text { do que puedan incorporar procesos para la generación } \\
\text { de conocimiento a través del trabajo intelectual que } \\
\text { realiza la fuerza laboral. }\end{array}$ & $\begin{array}{l}\text { (Balaz, 2004; Penco et al., 2020; Robertson \& O'Malley, } \\
\text { 2000). }\end{array}$ \\
\hline
\end{tabular}

Knowledge-based organisation

El conocimiento en las organizaciones como algo intan- $\quad$ (Seto, Arkison \& Williams, 2011; Nicolaescu et al., 2020; gible y abstracto, pero que es gestionado para competir. Škerlavaj, Dimovski \& Desouza, 2010).

Knowledge-intensive business

Empresas donde su principal activo es el conocimiento y el desarrollo óptimo de la investigación y desarrollo (Brenner, Capasso, Duschl, Frenken \& Treibich, 2018; (I\&D).

Knowledge-intensive business service

Servicios intensivos en conocimiento que requieren cons- (Basilio, Laredo \& Sanchez, 2019; Lee \& Miozzo, 2019; tante manejo de este para el desarrollo de su servicio Santos, 2020). mediante I\&D.

Knowledge-intensive companies

Compañías que crean conocimiento como valor para la $\quad$ (Afsar, Badir, Saeed \& Hafeez, 2017; Mathew, 2019).

resolución de problemas de sus clientes. 
Knowledge-intensive firm

Empresas que emplean el conocimiento de las personas para buscar y compartir soluciones intangibles en busca

de una ventaja competitiva.

Knowledge-intensive industries

Industrias con alto valor agregado que dependen en gran medida del conocimiento para el desarrollo de su objetivo final.

Knowledge-intensive organizations

Organizaciones cuyo objetivo principal es la explotación del conocimiento para su funcionamiento y comercialización.

Knowledge-intensive SMES

Pequeñas y medianas empresas donde el conocimiento es fundamental para su funcionamiento.

Fuente: elaboración de los autores.

Como se ilustra en la tabla 2, las etiquetas empleadas para el estudio de las orc no difieren de sus definiciones, sino del sustantivo. Este es el caso de 'organización', 'negocio', 'firma', 'industrias', 'compañía', etc., para hablar de tipo de organización; también aparece la forma de 'pyme' para distinguir según el tamaño; y, finalmente, etiquetas como 'basadas en conocimiento' versus 'intensivas en conocimiento' para situar la intensidad del conocimiento.

Para Starbuck (1992), en las oic el conocimiento es la entrada más importante y, por ello, el capital humano es dominante. La importancia del conocimiento presupone ciertos cambios con relación a la organización en general. Por ejemplo, los trabajadores se convierten en un factor crítico, debido a que en estos reposa el conocimiento (Alvesson, 1993; Blackler, 1993; Makani \& Marche, 2010; Miles, Belousova \& Chichkanov, 2018). De este modo, las oic son altamente dependientes de sus empleados (Alvesson, 1995; Greenwood, 2009; Kemp, Moerman \& Prieto, 2001; Robertson \& Swan, 2003; Sveiby, 1992), los cuales son altamente educados, cualificados y con habilidades (Alvesson, 1995; Deng, 2008; Ferdinan, 2013; Kuula, Haapasalo \& Tolonen, 2018), que permiten a la organización ofrecer soluciones creativas e innovadoras a problemas complejos del mercado (Alvesson, 1993, 1995; Blacker, 1995; Deng, 2008; Lee \& Miozzo, 2019). De acuerdo con esto, el conocimiento se convierte en valor agregado y la creatividad, en la llave de la productividad (Basilio, Laredo \& Sanchez, 2019; Makani \& Marche, 2012; Nurmi, 1998; Swart \& Kinnie, 2003). 
Existen algunas tipologías de oIC como las desarrolladas por Kemp et al. (2001) y Makani y Marche (2010). En Kemp et al. (2001), las orc se distinguen en dos tipos: livianas y pesadas. Para estos autores, las características de estos dos tipos de oic son:

- Los seres humanos (capital intelectual), identificados por la creatividad y la innovación, y altos niveles de educación con alto grado de profesionalización.

- Énfasis en las operaciones intensivas en conocimiento, las actividades básicas no se automatizan, los bienes materiales no son factores centrales.

- Relación con los clientes y los sistemas de prestación de servicios. Diferenciación en el valor real para el cliente.

- Dependencia hacia la lealtad del personal clave.

- Medición del éxito no solo por criterios financieros.

En la revisión de la literatura, se hallaron algunos tipos específicos de orc, entre las que se encuentran las organizaciones creativas (creative organizations). Según Winch y Schneider (1993), este tipo de organizaciones son contratadas para proporcionar soluciones innovadoras a problemas especiales, en los que se presentan procesos con altos niveles de incertidumbre en la tarea. Un ejemplo de este tipo de organizaciones son las asociaciones u organizaciones de arquitectos. Para responder a las demandas de sus clientes, hacen hincapié en la innovación y la resolución de problemas. De esta forma la creatividad se convierte en una ventaja competitiva (Wich \& Schneider, 1993).

Otro tipo de oIc es la organización profesional (professional organization). En este tipo de organizaciones, los servicios ofrecidos están regulados por una institución profesional. Por ejemplo, en las prácticas arquitectónicas, para poder diseñar un edificio, se requiere de un título profesional de un arquitecto, el cual está protegido por la ley, y para desarrollar este proceso es necesaria la supervisión de la institución profesional. En este sentido, los clientes acceden a un servicio intangible, del cual conocen tanto los procedimientos como las normas (Wich \& Schneider, 1993). Para Alvesson (1993), las organizaciones 
profesionales incluyen la noción de 'profesiones'. Es por ello que los ejemplos de este tipo de organizaciones son aquellas en las que se encuentran médicos, abogados, contadores, publicistas, arquitectos, informáticos, ingenieros, consultores de gestión y psicólogos.

Para las organizaciones de servicios (service organizations), lo predominante es la calidad del servicio basada en la experiencia en general, más que el producto final. Lo que hace especiales a este tipo de organizaciones es que los servicios son intangibles, así es heterogéneo en el que el rendimiento varía de un cliente a otro, y la producción y el consumo son inseparables. Como consecuencia, este tipo de organizaciones considera como sus principales activos a sus trabajadores y su reputación de ofrecer el servicio prometido (Dobrai \& Farkas, 2009; Wich \& Schneider, 1993).

Por último, las oic también han sido definidas en relación con su tamaño. Por ejemplo, Baptista, Annansingh, Eaglestone y Wakefield (2006), Bolisani, Dono y Scarso (2016) y Kozhevnikov (2019) estudian las pymes intensivas en conocimiento. Para estos autores, aunque los temas relacionados con la gestión del conocimiento han estado centrados en las grandes empresas, en las pymes se presentan procesos de innovación y gestión del conocimiento que sugieren la existencia de oIC de tamaños pequeños y medianos.

\subsection{Competencia y estrategia}

Los autores que analizan la competencia y la estrategia de las oic encuentran que el conocimiento es la principal estrategia para competir. La estrategia es única, rara, combinada, no imitable, explotable, basada en la innovación, en los recursos, en la creatividad y en recursos intangibles, y proviene principalmente de los trabajadores (Alvesson, 2011; Filstad \& Gottschalk, 2009; Kärreman, 2010; Nurmi, 1998; Swart \& Kinnie, 2003). De esta forma es posible identificar el vínculo existente entre conocimiento-trabajador como generador de las estrategias.

Las orc son altamente dependientes de la lealtad de sus empleados. Esto se debe a que sus empleados tienen un alto grado de profesionalismo, son altamente educados y son expertos creativos (Kemp, Moerman \& Prieto, 2001; Makani \& Marche, 2010, 2012; Sveiby, 1992). Esta dependencia confirma la idea de que, para estas organizaciones, a diferencia de las organizaciones tradicionales, el conocimiento es un factor crítico y más importante 
que otras entradas (inputs). Es por ello que las oIc compiten con este elemento, el cual es considerado como creatividad e innovación (Brotherton, 1998; Doloreux, Shearmur \& Rodriguez, 2018; Greenwood, 2009; Makani \& Marche, 2010, 2012; Morten \& Syvertsen, 1998; Song, Zhang \& Shi, 2007; Sveiby, 1992).

Las OIC atienden a sus clientes individualmente, quienes tienen problemas complejos y son resueltos gracias a la aplicación del conocimiento, lo que genera valor agregado (Kemp, Moerman \& Prieto, 2001; Sveiby, 1992). Dado que la estrategia utilizada es única y difícil de imitar, le permite la creación de productos intensivos en conocimiento y competir en la economía o sociedad del conocimiento (Greenwood, 2009; Kemp, Moerman \& Prieto, 2001; Mol \& Brandl, 2018).

Al ser el conocimiento la fuente de ventaja competitiva para la oic, la literatura de este campo involucra en la mayoría de sus publicaciones las prácticas de gestión del conocimiento. Esto se debe a que las oic requieren que el conocimiento se distribuya por toda la organización, de modo que cualquier persona pueda acceder a él. Ello contrasta con las organizaciones tradicionales, en las que el conocimiento se concentra en la cúspide de la estructura organizacional (Kärreman, Sveningsson \& Alvesson, 2002). De acuerdo con Kemp et al. (2001), la estrategia organizacional para las oIc se caracteriza por siete requerimientos: 1) la necesidad de gestionar las capacidades humanas de una manera estratégica y holística; 2) trabajar en red con socios internos y externos; 3) crear estructuras organizacionales adaptativas e interactivas; 4) balancear la eficiencia de los procesos versus la innovación; 5) balancear la motivación individual y de los grupos; 6) equilibrar los flujos de fuerzas de arriba hacia abajo y de abajo hacia arriba; y 7) desarrollar un estilo competitivo y sostenible.

Kärreman (2010) sostiene que en las orc la ventaja competitiva no consiste en la mano de obra barata o en las económicas de escala, sino en la aplicación de conocimientos. Estas ideas también son trabajadas en Alvesson (2011) y Makani y Marche (2012), quienes utilizan esta aproximación del conocimiento como fuente de ventaja competitiva para diferenciar a las oIc de las organizaciones tradicionales. 


\subsection{Conocimiento}

Para autores como Alvesson (1993, 2000, 2011), Greenwood (2009) y Makani y Marche (2010), la definición de las oic ha sido difícil debido a que es ambiguo y confuso definir conocimiento, y porque no existe consenso entre académicos y profesionales sobre lo que significa intensidad de este. Sin embargo, en la literatura correspondiente a las oIC existen ciertas consideraciones sobre lo que este concepto significa.

Nonaka y Takeuchi (1999) clasifican el conocimiento humano en dos tipos. Por una parte, el conocimiento explicito, que se expresa a través del lenguaje formal, enunciados gramaticales, expresiones matemáticas, especificaciones, manuales, etc. Esta clase de conocimiento puede transmitirse fácilmente de un individuo a otro. Por otra parte, el conocimiento tácito, que resulta difícil de enunciar mediante el lenguaje formal, debido a que se refiere a lo aprendido gracias a la experiencia personal e involucra factores tangibles como las creencias, el punto de vista propio y los valores. Dicho tipo de conocimiento no se ha tomado en cuenta como lo que es: "Un componente fundamental del comportamiento humano" (Nonaka \& Takeuchi, 1999, p. xiii).

En la literatura se registran diversas consideraciones con respecto al término conocimiento. Entre ellas, la más citada es la del conocimiento esotérico (Alvesson, 1993, 2001, 2011; Blacker, 1993; Blackler, 1995; Ferdinand, 2013; Kärreman, 2010; Makani \& Marche, 2010, 2012; Tuominen \& Martinsuo, 2019; Starbuck, 1992; Swart \& Kinnie, 2003). Este término hace alusión a la experticia de los trabajadores de las oIc. De esta forma, el conocimiento es un elemento de difícil acceso, secreto e incomprensible para quienes no están en altos niveles de experticia, lo que diferencia del conocimiento común (Estrada-Torres et al., 2019; Starbuck, 1992; Kärreman, 2010). Como consecuencia, del conocimiento se derivan productos únicos y poco imitables para la competencia (Nurmi, 1998). Autores como Blacker (1993) han considerado que el conocimiento esotérico es necesario para el logro de este tipo de organización, el cual se encuentra íntimamente ligado con la gestión. Por su parte, Starbuck (1992) señala que el conocimiento en las oIc se convierte en experiencia esotérica, la cual genera poder monopólico en los mercados. Esta experiencia no solo se compone de conocimiento, sino también de habilidades simbólicas y analíticas de los empleados (Alvesson, 2011). 
Otra de las consideraciones más frecuentes es el conocimiento como valor agregado. Dado que este tipo de organizaciones poseen conocimiento esotérico, es posible ofrecer valor agregado al mercado a través de bienes y servicios orientados a satisfacer las necesidades complejas de clientes y consumidores (Alvesson, 1993, 1995, 2001; Blacker, 1995; Brotherton, 1998; Garri, 2020; Nurmi, 1998; Sveiby, 1992; Winch \& Shneider, 1993).

El conocimiento es también entendido como activo intangible. Conforme con Song, Zhang y Shi (2007), el conocimiento en las orc es capital intelectual y activo intangible, el cual se combina con una alta complejidad de interfaces, que incluyen tecnología, capital humano, patentes, marcas y rutinas organizacionales. Knigths, Murray y Willmott (1993) señalan que los trabajadores del conocimiento proporcionan activos y recursos intangibles que se convierten vitales para el éxito competitivo sostenido y el crecimiento. En este sentido, Swart y Kinnie (2003) indican que el capital intelectual y los activos intangibles asociados al conocimiento son una característica fundamental de las oic. Para estos autores, los activos intangibles generan ingresos, gracias a la naturaleza del despliegue del conocimiento que poseen los trabajadores, es decir, gracias a que extienden la promulgación de conocimiento tácito en circunstancias nuevas (Swart \& Kinnie, 2003).

El capital intelectual es otro elemento asociado con el conocimiento. Para Brotherton (1998), el conocimiento es el factor esencial para sostener a las organizaciones. Según este autor, la clave del éxito se configura, además de en una estrategia para desarrollar la base de conocimientos de la organización, con el desarrollo del capital intelectual. Por su parte, Baptista et al. (2006) consideran que el conocimiento tácito es la base del capital intelectual de las oic, el cual necesita ser expresado y administrado. Song et al. (2007) señalan el conocimiento como capital intelectual, el cual es un objeto que pertenece al mundo exterior y a la tarea del cerebro.

El último de los elementos asociados al conocimiento encontrado en la revisión de la literatura es el relacionado con el conocimiento como una fuente de ventaja competitiva. La noción de conocimiento en Starbuck (1992) anota este elemento como un recurso, que es complejo y difícil de tratar, sin embargo, para este autor, el conocimiento constituye el recurso más importante del que dispone una empresa para la ventaja competitiva. En 
este sentido, Filstad y Gottschalk (2009), bajo la teoría basada en conocimiento (knowledge-based theory), sostienen que el conocimiento constituye el principal recurso tanto para el éxito como para la sobrevivencia de las organizaciones.

\subsection{Contexto}

La literatura con referencia a las orc considera que estas se encuentran en el marco de la sociedad o economía del conocimiento, la cual se caracteriza por la transición de la gestión de personas, la flexibilidad y libertad máxima de las acciones empresariales, la creencia en la incertidumbre por los mercados desregulados para optimizar las inversiones de capital y la formación de una economía interconectada en tiempo real (Ferraz \& Pereira, 2018; Krüger, 2006; Naisbitt, 1983). La economía del conocimiento es una de las características más discutidas en la literatura sobre el contexto de las orc. Esta es relevante para comprender las oic debido a que condiciona sus características, generando cambios en su interior, a partir de las nuevas demandas y exigencias del mercado (Alvesson, 2011; Kemp et al., 2001; Sveiby, 1992; Mohammadi, 2019).

Para autores como Hertog y Bilderbeek (2000), en la economía del conocimiento, el recurso clave es el mismo "conocimiento". Según estos autores, existen dos razones: la primera es que la competitividad de la mayoría de las industrias depende de la creación de conocimiento innovador y la transformación de procesos. La segunda es que la intensidad del conocimiento en las industrias contribuye al crecimiento económico y a la competitividad de los países. En este sentido, Lundvall y Borrás (1997) sostienen que la globalización no solo aumentó la competencia del mercado, sino que la transformó en una competencia basada en el conocimiento y en el aprendizaje. Por su parte, para Drucker, Dyson, Handy, Saffo y Senge (1997), la contribución más importante de la administración que se debe hacer en el siglo xxi es aumentar la productividad del trabajo del conocimiento y el conocimiento de los trabajadores. Conforme con ellos, el activo más valioso de una organización en este contexto es el conocimiento de los trabajadores y su productividad.

Otras características que resalta la literatura sobre el contexto de las oic son el posindustrialismo; el mercado creciente de los servicios profesionales, y, por ello, del sector servicios; la sociedad informática o economía de la información o servicios, etc. (Feser \& Proeger, 2018). Finalmente, las investigaciones más recientes vinculan las oic en el marco 
de la Cuarta Revolución Industrial, en la que el conocimiento posibilita la inteligencia artificial, la automatización, el uso intensivo de la tecnología y las máquinas, y la virtualización de los procesos productivos (Kozhevnikov, 2019; Lee \& Miozzo, 2019; Mathew, 2019; Wyrwich, 2019).

\subsection{Estructura}

Autores como Alvesson (2000) señalan que en las oIc existe una fuerte dependencia de la libre determinación, pues se le resta importancia a la jerarquía dimensional, ya que surge la necesidad de una amplia comunicación para la coordinación y solución de problemas. De esta forma, se considera que las oic se salen de los principios burocráticos, lo que genera una ruptura en el paradigma de la jerarquía y el control (Grey \& Sturdy, 2009; Kärreman, Sveningsson \& Alvesson, 2002; Mattarelli, Bertolotti \& Incerti, 2015; Mathew, 2019).

En estas organizaciones, los empleados asumen una cultura de autocontrol, en la cual sin la necesidad de la supervisión hacen lo que deben hacer. Adicional a ello, el conocimiento deja de estar en las máquinas y procesos estandarizados para estar en las personas, en sus capacidades y habilidades. Como resultado, se habla de una desburocratización en la que no existen normas estrictas, estándares fijos para medir el progreso y procesos de esta naturaleza. La división del trabajo también desaparece y se promueve el compartir el conocimiento y el trabajo en equipo (Grey \& Sturdy, 2009).

Según Starbuck (1992), en las oic la coordinación se da por medio de recompensas y normas sencillas:

- La importancia de cada individuo como experto le acredita cierta autonomía, un control fuerte lo ahuyentaría.

- Algunas de las oic derivan sus valores de las universidades, en la cual cada uno sabe qué hacer, cuándo y dónde.

- Los expertos trabajan de manera independiente, en los diferentes proyectos solo incluyen a unas pocas personas. 
- La inestabilidad de los proyectos y servicios, causada por las variaciones en las demandas, genera que en las oIc se necesite la flexibilidad en la estructura organizacional.

Para Sydánmaanlakka (2002), las oIc adoptan la adhocracia como estructura organizacional, estructura flexible para servir mejor a los objetivos de la organización. Autores como Kemp et al. (2001) y Makani y Marche (2010, 2012) también consideran que las estructuras de las oic son en redes, lo que permite mayor flujo de comunicación para toda la organización, es decir, mayor flujo del conocimiento. Este permite el desarrollo de los procesos relacionados con el conocimiento, entre los que se destaca el aprendizaje organizacional.

La literatura discute principalmente la estructura organizacional de las oic como estructuras poco jerarquizadas y desburocratizadas. Cabe resaltar que existen otros adjetivos y características de estas, como estructuras por proyectos, descentralizadas y basadas en tecnología (Grey \& Sturdy, 2009; Mattarelli et al., 2015; Mathew, 2019; Starbuck, 1993; Winch \& Shneider, 1993).

Las estructuras poco jerarquizadas y descentralizadas permiten mayor autonomía de los empleados, no solo en la toma de decisiones, sino además en la organización del tiempo de trabajo. Es por ello que el teletrabajo, el trabajo parcial y a distancia permiten que los empleados desarrollen sus actividades y cumplan con sus responsabilidades sin la necesidad de estar de manera presencial en su puesto de trabajo (Mathew, 2019; Wyrwich, 2019).

\subsection{Procesos y productos}

Las OIC se enfatizan en las operaciones intensivas en conocimiento, en la automatización por medio de inteligencia artificial y en el uso intensivo de la tecnología para algunos de sus procesos, como la atención de clientes y la fabricación de ciertos bienes. Sin embargo, cuando son servicios, estos se caracterizan por ser personalizados y no siempre automatizados. Así mismo, los bienes materiales dejan de ser un factor central y se da paso a la innovación, creatividad, etc. La literatura en su mayoría refleja un especial interés por la resolución de problemas complejos. Esto se debe a que en el mercado los requerimientos de los clientes y consumidores son cada vez más complejos y diversos (Alvesson, 1993, 1995; Brotherton, 
1998; Robertson \& Swan, 2003; Liu, Lattemann, Xing \& Dorawa, 2019). Adicional a ello, este tipo de organizaciones solucionan de una forma creativa, lo que hace que sus clientes obtengan un mayor valor agregado y productos difíciles de encontrar en la competencia (Alvesson, 1993; Brotherton, 1998; Deng, 2008; Lönnqvist, 2005; Zhang, 2019).

Según la literatura, los procesos se centran en la solución creativa de problemas, los cuales se caracterizan por ser complejos y requerir de la utilización intensiva del conocimiento. Como resultado de los procesos que realizan las oIc, sus productos (bienes y servicios) son en esencia de conocimiento (Zhou, Kautonen, Wang \& Wang, 2017). Las principales características asociadas a los productos son la cualificación, que no son imitables, intangibles y diversificados. De acuerdo con Alvesson (2011), las orc producen resultados excepcionalmente buenos con la ayuda de expertos en circulación. Para ello son importantes los recursos que otorga el conocimiento, lo que incluye la tecnología, la confianza del cliente, la imagen corporativa, la gestión de la logística, la cultura organizacional y la gestión de conocimientos (Kozhevnikov, 2019; Sokeila et al., 2003).

Los productos o derivados de las actividades de generación de conocimiento de las oic están asociados específicamente con el tipo de orc. Conforme con su naturaleza y orientación, es posible identificar qué tipo de conocimiento producen y cuáles son los problemas que pueden solucionar con él (Kozhevnikov, 2019; Zhou et al., 2017). En el apartado de "Etiquetas y tipos de oic", se encuentran algunos ejemplos de ello.

Así mismo, el uso intensivo del conocimiento y el avance tecnológico ha revolucionado el mercado de los servicios, por lo que los constantes cambios hacen compleja la categorización de los productos del conocimiento. No obstante, la producción en el marco de la Cuarta Revolución Industrial ha posibilitado la combinación de la realidad física con la virtual, la combinación del conocimiento humano con el de las máquinas y el uso de robots, las interacciones entre empresa y consumidores, y el acceso a servicios de manera virtual y presencial. 


\subsection{Trabajadores}

Debido al trabajo de naturaleza intelectual que se presenta en las orc, los trabajadores adquieren características específicas que los diferencian de los de organizaciones tradicionales. Una de las principales características de los trabajadores de la oic es la alta educación. Aunque ciertas investigaciones han encontrado que en estas organizaciones los empleados llegan a alcanzar títulos de doctorado $(\mathrm{PhD})$, también poseen una vasta experiencia que los hace expertos en diferentes áreas del conocimiento. Es por ello que la literatura habla de trabajadores del conocimiento, que son en su mayoría expertos, profesionales, especialistas, creativos y calificados, que gracias a su compromiso y orientación no requieren de supervisión, lo que los hace independientes (Alvesson, 1995, 2000, 2001; Baptista et al., 2006; Berraies \& Bchini, 2019; Deng, 2008; Makani \& Marche, 2010, 2012; Starbuck, 1992; Sveiby, 1992; Winch \& Shneider, 1993).

Para Starbuck (1992), los expertos son diferentes a los profesionales, dado que son personas con educación formal y experiencia equivalente a un título de doctorado, y en las oic los expertos son al menos una tercera parte del personal. De acuerdo con Winch y Schneider (1993), lo que diferencia a las orc de otras organizaciones es el empleo de este tipo de personal, el cual comercializa de forma independiente y en gran medida con clientes corporativos. Como consecuencia, Swart y Kinnie (2003) sostienen que el éxito de las orc se obtiene a partir de la ventaja competitiva del capital humano y social que conforman sus activos.

Las características de los empleados refieren al concepto de trabajo del conocimiento. Según Knights, Murray y Willmot (1993), este concepto está asociado con la actividad de los que ocupan una posición privilegiada dentro de la división del trabajo. Para estos autores, los trabajadores del conocimiento son individuos altamente calificados que pertenecen o forman un componente distinto de un grupo de empleados élites, profesionales y directivos. El monopolio de la destreza de esta élite de profesionales altamente calificados, excepcionalmente inteligente o individuos que trabajan duro, se asocia con el poder de diseñar o controlar su propio trabajo, y de concebir y delegar otras tareas que requieren relativamente de poca o ninguna experiencia (Millar, Chen \& Waller, 2017; Knigths, Murray \& Willmot, 1993; MacGillivray, 2018). 
Los nuevos mercados de consumo y la demanda de productos, así como las transformaciones propias de la forma en que se organizan las oIc y desarrollan sus actividades productivas, han generado que algunos trabajos sean reemplazados por la máquina y plataformas tecnológicas. No obstante, este fenómeno también ha ocasionado que las empresas requieran de nuevos tipos de trabajos, principalmente aquellos relacionados con el desarrollo de sistemas, aplicaciones digitales, arquitectura virtual, robótica, etc. Esto otorga la importancia de la I\&D para las organizaciones, y conocimientos centrados en la experticia (Kozhevnikov, 2019; Lee \& Miozzo, 2019).

\section{Discusión}

La revisión de la literatura permite identificar algunos cambios y tendencias en la investigación y conceptualización de las oic. Desde las primeras publicaciones hasta el año 2019, se evidencia que la mayor producción académica de artículos relacionados con este tema se concentra en los últimos siete años (56\%). Esta tendencia da cuenta de un interés académico por el aumento de este tipo de organizaciones en el contexto económico y empresarial. Durante el período analizado de la literatura, se encuentra que en los primeros años de publicación (período de incubación: 1992-2004) las orc se examinan principalmente a la luz de la etiqueta knowledge-intensive firm, para luego abordarse desde 2005 hasta 2009 bajo la etiqueta knowledge-intensive business service, lo que refleja el crecimiento del sector servicio en el contexto internacional.

Las investigaciones de este tema han estado lideradas por autores del contexto europeo (58\%), seguido del contexto norteamericano (26\%). Igualmente, las investigaciones se caracterizan por abordarse desde enfoques cualitativos (57\%), y concentrarse en describir principalmente la noción de 'conocimiento' (41\%) como el principal atributo de las oic y el factor diferenciador con otro tipo de organizaciones. No obstante, para diversos autores, las organizaciones son cada vez más dependientes del conocimiento, y esto se asocia con las características del actual contexto empresarial y de las economías mundiales. 
Las características observadas en la literatura académica con relación a las oic permiten identificar que este tipo de organizaciones se diferencian de las organizaciones tradicionales por la incorporación del conocimiento en todas sus dimensiones, por lo que su éxito en la gestión y resultados organizacionales se debe a la generación y aplicación de los conocimientos. Ello implica que tanto la organización del trabajo como el tipo de trabajadores y sus productos giren en torno a la gestión del conocimiento. Debido a que el conocimiento emerge de los trabajadores, hace que estas organizaciones sean altamente dependientes de ellos, considerándolos como lo más importante. En este sentido, el capital humano o capital intelectual viene a configurar un factor dominante, y la naturaleza intelectual del trabajo se materializa con el uso sofisticado del conocimiento, que permite generar innovaciones y creatividad (Alvesson, 1993, 2000; Baptista et al., 2006; Deng, 2008; Grey \& Sturdy, 2009; Kemp et al., 2001; Makani \& Marche, 2010, 2012; Yalabik, Swart, Kinnie \& Van Rossenberg, 2017).

Al ser el conocimiento el núcleo de gestión de las orc, no solo los trabajadores se posicionan como el factor más importante de ellas, sino que los activos físicos como las máquinas y otros recursos empiezan a ser considerados como relevantes de manera secundaria. Para la generación de conocimiento se requiere de formas específicas de estructuras jerárquicas que se caracterizan principalmente por ser flexibles, adaptables, en red o matriciales, y con bajos niveles jerárquicos. Además, los trabajadores desempeñan trabajos de conocimiento que son complejos, pero con mayor autogestión, toma de decisión y autoridad. Esto desdibuja la idea de las organizaciones tradicionales en donde el conocimiento se encuentra concentrado en los cargos de toma de decisión de la cúspide de la jerarquía organizacional.

El conocimiento que es especializado, esotérico y difícil de imitar permite a las oic producir bienes y servicios mediante una relación personalizada y estrecha con sus clientes, proveedores y socios estratégicos. De la misma forma, en la literatura se encuentra que la comprensión de los clientes posibilita a las oic evaluar la evolución y las transformaciones de las demandas de los mercados. De este modo, las estrategias pueden ser tanto reactivas como proactivas.

La importancia del conocimiento configura la gestión en este tipo de organizaciones, pues determina las demás características definidas, como la forma de competir y formular 
estrategias, el modo de organizar el trabajo y los cargos (estructura jerárquica), el tipo de productos (principalmente servicios y tecnología) y el tipo de trabajadores. Este fenómeno implica prácticas como las de la gestión del conocimiento, las cuales constituyen un elemento estratégico que abarca todas las dimensiones de las empresas y se articula con las prácticas de recursos humanos.

Si bien la literatura ofrece elementos teóricos y empíricos sobre algunas de las características de las oic, el actual contexto de los negocios y las organizaciones hace que las organizaciones tradicionales, o concentradas en industrias manufactureras y productivas, sean dependientes cada vez más del conocimiento, por lo que las organizaciones del siglo XXI son organizaciones de conocimiento, y sus diferencias varían en la intensidad del conocimiento para la producción de sus bienes y servicios, sus estrategias competitivas y la institucionalización de la gestión del conocimiento vinculada a un tipo de trabajador como conocimiento experto y de alto nivel académico, que desempeña sus labores con alta relación con la tecnología, lo que permite responder a unas demandas cambiantes y complejas del mercado.

\section{Conclusiones}

Este artículo se centró en identificar las características de las oic a partir de una revisión sistemática en la literatura. Como se ha señalado, este tipo de organizaciones configuran procesos productivos y comerciales en la llamada sociedad o economía del conocimiento, pero que se empiezan a examinar como objeto de investigación académico en el marco de la Cuarta Revolución Industrial. Los resultados hallados permiten identificar características de las oIc, diferenciándolas de las organizaciones tradicionales, que desde el campo organizacional se han definido en el contexto industrial, posindustrial, y con el cambio hacia la sociedad del conocimiento. En los artículos analizados se resaltan algunos factores diferenciadores de las orc, como el conocimiento esotérico, la alta formación de los trabajadores, el trabajo de naturaleza intelectual, la solución creativa de problemas complejos, la estructura poco jerarquizada, la economía del conocimiento y la competencia del conocimiento. No obstante, es importante 
reconocer que si bien durante la última década las publicaciones sobre este tema han aumentado y se evidencia un interés académico, también sugieren que cada vez las organizaciones en general han incorporado el conocimiento como núcleo de su quehacer y funciones, lo que configura el término de 'organización intensiva del conocimiento' como una sombrilla bajo la cual se pueden examinar distintos tipos de organizaciones (e.g., privadas, públicas, del tercer sector, productivas, de la académica, industrias, multinacionales, pymes).

Dicha incorporación implica una transformación tanto en las prácticas administrativas como en la forma en que se organiza el trabajo y las funciones organizacionales, entre las que se destacan la gestión del conocimiento, el aprendizaje organizacional, los enfoques de la innovación, en donde el conocimiento es transversal a los procesos de producción, de gestión humana, de comercialización, y lo que esta noción representa para los empleados, accionistas y clientes. Esto desdibuja el modo en que las organizaciones de la sociedad industrial, posindustrial e informática eran gestionadas y se caracterizaban con variables como sus estructuras, sus productos y servicios, el tipo de trabajadores, la definición de estrategias.

El ejercicio que sustenta el presente trabajo supone un avance teórico en el campo de la gestión del conocimiento y de las organizaciones de conocimiento, teniendo en cuenta que no existen definiciones en la literatura que abarquen en amplitud las características de las orc. Se debe tener en cuenta que las definiciones utilizadas como fuentes de información provienen de autores principalmente de Norteamérica y Europa, por lo que es posible que a la hora de las comparaciones las orc en los contextos latinoamericanos presenten ciertas tensiones con los resultados reportados.

La caracterización de las oic propuesta en este artículo permite tener una perspectiva más amplia sobre lo que son este tipo de organizaciones. Algunas investigaciones como las de Makani y Marche (2010) establecen una tipología de orc a partir de ciertos factores, como el tipo de trabajador y la estructura en redes, lo que se amplió con el presente estudio, teniendo en cuenta no solo su naturaleza, sino también su contexto, la estrategia y la noción de conocimiento. Adicional a ello, se revisaron más documentos que se han publicado sobre el tema, extendiendo el estado del arte hasta 2020. 
A partir de la caracterización de las oic se contribuyó a un marco analítico y teórico del tema, el cual permite a futuro el desarrollo de propuestas de modelos de gestión y formulación de estrategias, ya que estos se originan desde las características específicas de las organizaciones. Esto posibilita también las prácticas de gestión del conocimiento, las cuales son un núcleo central para este tipo de organizaciones. En adición, este texto sirve de fundamento teórico o de punto de partida para futuras investigaciones empíricas que busquen identificar la naturaleza de las oic y sus prácticas en contextos específicos.

Si bien el estudio se centró en publicaciones de catálogos digitales que suponen calidad y actualidad de estas, es posible que estudios realizados en Colombia y en países de la región se hayan quedado por fuera, invisibilizando las características y el contexto de las orc de esta región, que podrían diferenciarse del de otras regiones del mundo. Así mismo, es esencial resaltar que pueden existir muchas más publicaciones que podrían enriquecer los hallazgos y discusiones del presente artículo, pero que por decisiones de los autores no se publicaron en las revistas de los catálogos digitales seleccionados metodológicamente para el estudio. Es por ello que para futuras investigaciones se sugiere incorporar otros catálogos digitales, entre ellos los de acceso abierto que caracterizan el ejercicio de publicaciones de la región de América Latina y el Caribe.

\section{Referencias}

Afsar, B., Badir, Y. F., Saeed, B. B., \& Hafeez, S. (2017). Transformational and transactional leadership and employee's entrepreneurial behavior in knowledge-intensive industries. International Journal of Human Resource Management, 28(2), 307-332. https://doi.org /10.1080/09585192.2016.1244893

Aktouf, O. (2011). La metodología de la investigación en las ciencias sociales y el enfoque cualitativo en las organizaciones. Una introducción al procedimiento clásico y una crítica. Cali: Universidad del Valle.

Alvesson, M. (1993). Organizations as rhetoric: knowledge-intensive firms and struggle with ambiguity. Journal of Management Studies, 30(6), 997-1015.

Alvesson, M. (1995). Management of knowledge-intensive companies. Berlin: Walter de Gruyter \& Co. 
Alvesson, M. (2000). Social identity and the problem of loyalty in knowledge-intensive companies. Journal of Management Studies, 37(8), 1101-1123.

Alvesson, M. (2001). Knowledge work: ambiguity, image and identity. Human Relations, $7(54), 863-886$.

Alvesson, M. (2011). De-essentializing the knowledge intensive firm: reflections on sceptical research going against the mainstream.Journal of Management Studies, 48(7), 1640-1661.

Balaz, V. (2004). Knowledge-intensive business services in transition economies. The Service Industries Journal, 24(4), 83-100.

Banco Mundial. (2019). Comercio de servicios. Recuperado de https://datos.bancomundial. org/indicador/BG.GSR.NFSv.GD.zS

Baptista, M., Annansingh, F., Eaglestone, B., \& Wakefield, R. (2006). Knowledge management issues in knowledge-intensive sMEs. Journal of Documentation, 6(1), 101-119.

Basilio, O., Laredo, P., \& Sanchez, P. (2019). The organization of R\&D activities in large knowledge intensive business services: the case of a "big four" consultancy. International Journal of Innovation Management, 23(3). https://doi.org/10.1142/S1363919619500270

Berraies, S., \& Bchini, B. (2019). Effect of leadership styles on financial performance: mediating roles of exploitative and exploratory innovations case of knowledge-intensive firms. International Journal of Innovation Management, 23(3). https://doi.org/10.1142/ S1363919619500208

Blackler, F. (1993). Knowledge and the theory of organizations: organizations as activity systems and the reframing of management. Journal of Management Studies, 30(6), 863-884.

Blackler, F. (1995). Knowledge, knowledge work and organizations: an overview and interpretation. Organization Studies, 6(16), 1021-1046.

Blackler, F., Reed, M., \& Whitaker, A. (1993). Editorial introduction: knowledge workers and contemporary organizations. Journal of Management Studies, 30(6), 851-862.

Bolisani, E., Dono, A., \& Scarso, E. (2016). Relational marketing in knowledge-intensive business services: an analysis of the computer services sector. Knowledge Management Research \& Practice, 110. http://doi.org/10.1057/kmrp.2014.35

Braga, A., Marques, C. S., \& Serrasqueiro, Z. (2018). Internationalisation strategy of knowledge-intensive business services. Journal of the Knowledge Economy, 9(2), 359-377. https:// doi.org/10.1007/s13132-017-0461-5

Brenner, T., Capasso, M., Duschl, M., Frenken, K., \& Treibich, T. (2018). Causal relations between knowledge-intensive business services and regional employment growth. Regional Studies, 52(2), 172-183. https://doi.org/10.1080/00343404.2016.1265104 
Brotherton, B. (1998). Developing a culture and infrastructure to support research-related activity in further education institutions: a knowledge-based organisations perspective. Research in Post-Compulsory Education, 3(3), 311-328.

Castellani, P., Rossato, C., Giaretta, E., et al. (2020). Tacit knowledge sharing in knowledge-intensive firms: the perceptions of team members and team leaders. Review Management Science, 15, 125-155. https://doi.org/10.1007/s11846-019-00368-x

Deloitte Insights. (2019). The Fourth Industrial Revolution in here. Are you ready? Available from https://www2.deloitte.com/cn/en/pages/consumer-industrial-products/articles/industry-4-0-technology-manufacturing-revolution.html

Deng, P. (2008). Applying a market-based approach to the development of a sharing-enabled км model for knowledge-intensive small firms. Information Systems Management, 25, 174-187.

Ditillo, A. (2004). Dealing with uncertainty in knowledge-intensive firms: the role of management control systems as knowledge integration mechanisms. Accounting, Organizations and Society, 29, 401-421.

Dobrai, K., \& Farkas, F. (2009). Knowledge-intensive business services: a brief overview. Perspectives of Innovations, Economics \& Business, 3, 15-17.

Doloreux, D., Shearmur, R., \& Rodriguez, M. (2018). Internal R\&D and external information in knowledge-intensive business service innovation: complements, substitutes or independent? Technological and Economic Development of Economy, 24(6), 2255-2276. https:// doi.org/10.3846/tede.2018.5694

Drucker, P. F. (2002). La gerencia: tareas, responsabilidades y prácticas. Argentina: El Ateneo.

Drucker, P., Dyson, E., Handy, C., Saffo, P., \& Senge, P. (1997). Looking ahead: implications of the present. Harvard Business Review, 75(5), 18-32.

Estrada-Torres, B., Richetti, P. H. P., Del-Río-Ortega, A., Baião, F. A., Resinas, M., ... Ruiz-Cortés, A. (2019). Measuring performance in knowledge-intensive processes. ACM Transactions on Internet Technology, 19(1). https://doi.org/10.1145/3289180

Ferdinand, J. (2013). Custom Liverpool managing knowledge. Stroud, United Kingdom: Palgrave Macmillan.

Ferraz, D. E., \& Pereira, E. T. (2018). The economic role of small knowledge intensive firms in European member states. International Journal of Knowledge-Based Development, 9(3), 221-243. https://doi.org/10.1504/IJKBD.2018.094898

Feser, D., \& Proeger, T. (2018). Knowledge-intensive business services as credence goods. A demand-side approach. Journal of the Knowledge Economy, 9(1), 62-80. https://doi. org/10.1007/s13132-015-0320-1 
Filstad, C., \& Gottschalk, P. (2009). How knowledge organizations work: the case of real estate agencies. International Real Estate Review, 12(1), 88-97.

Fink, A. (2005). Conducting research literature review: from paper to the internet. London: SAGE.

Garri, M. (2020). Coopetition, value co-creation, and knowledge-enhancement in the UK alpaca industry: a multi-level mechanism. European Management Journal. https://doi. org/10.1016/j.emj.2020.11.001

Greenwood, D. J. (2009). Are research universities knowledge-intensive learning organizations? In D. Jemielniak \& J. Kociatkiewicz (Eds.), Handbook of research on knowledge-intensive organizations (pp. 1-18). Hershey, PA: Information Science Reference.

Grey, C., \& Sturdy, A. (2009). Historicising knowledge-intensive organizations: the case of Bletchley Park. Management \& Organizational History, 4(2), 131-150.

Herstad, S. J., \& Ebersberger, B. (2015). On the link between urban location and the involvement of knowledge-intensive business services firms in collaboration networks. Regional Studies, 49(7), 1160-1175. http://doi.org/10.1080/00343404.2013.816413

Hertog, P., \& Bilderbeek, R. (2000). The new knowledge infraestructure: the role of technology-based knowledge-intensive business services in national innovation systems. In M. Boden \& I. Miles (Eds.), Services and the knowledge-based economy (pp. 222-264). London: Routledge.

Horváth, K., \& Berbegal-Mirabent, J. (2020). The role of universities on the consolidation of knowledge-based sectors: a spatial econometric analysis of kIBs formation rates in Spanish regions. Socio-Economic Planning Sciences, 1-11. https://doi.org/10.1016/j. seps.2020.100900

Kärreman, D. (2010). The power of knowledge: learning from 'learning by knowledge-intensive firm'. Journal of Management Studies, 47(7), 1405-1416.

Kärreman, D., Svenningsson, S., \& Alvesson, M. (2002). The return of the machine bureaucracy? Management control in the work settings of professionals. International Studies of Management \& Organization, 32(2), 70-93.

Kemp, J., Moerman, P., \& Prieto, J. (2001). On the nature of knowledge-intensive organisations: strategy and organisations in the new economy. In $7^{\text {th }}$ International Conference on Concurrent Enterprising.

Knights, D., Murray, F., \& Willmott, H. (1993). Networking as knowledge work: a study of strategic interorganizational development in the financial services industry. Journal of Management Studies, 30(6), 975-995. 
Kozhevnikov, M. V. (2019). A transition to knowledge-intensive service activities in power industry: a theoretical framework. ШІт Transactions on Ecology and the Environment, 222, 13-25. https://doi.org/10.2495/EQ180021

Krüger, K. (2006). El concepto de sociedad del conocimiento. Biblio 3 W. Revista bibliográfica de geografía y ciencias sociales, 11(683).

Kuivalainen, O., Sundqvist, S., Puumalainen, K., \& Cadogan, J. (2004). The effect of environmental turbulence and leader characteristics on international performance: are knowledge-based firms different? Canadian Journal of Administrative Sciences, 21(1), 35-50.

Kuula, S., Haapasalo, H., \& Tolonen, A. (2018). Cost-efficient co-creation of knowledge intensive business services. Services Business, 12, 779-808.

Kvaløy, O., \& Olsen, T. E. (2008). Cooperation in knowledge-intensive firms. Journal of Humana Capital, 2(4), 410-440.

Lee, H., \& Miozzo, M. (2019). Which types of knowledge-intensive business services firms collaborate with universities for innovation? Research Policy, 48(7), 1633-1646.

Liu, Y., Lattemann, C., Xing, Y., \& Dorawa, D. (2019). The emergence of collaborative partnerships between knowledge-intensive business service (KIBs) and product companies: the case of Bremen, Germany. Regional Studies, 53(3), 376-387. https://doi.org/10.1080/00 343404.2018.1510178

Lönnqvist, A. (2005). Business performance measurement for knowledge-intensive organizations. In S. S. Kambhammettu (Ed.), Business performance measurement: intellectual capital-valuation models (pp. 17-35). India: Le Magnus University Press.

Lundvall, B. (1999). La base del conocimiento y su producción. Eknomiaz Revista Vasca de Economía, (45), 14-37.

Lundvall, B., \& Borrás, S. (1997). The globalising learning economy: Implications for innovation policy. Brussels, Belgium: DG XII, Comission of the European Union.

MacGillivray, A. E. (2018). Leadership as practice meets knowledge as flow: emerging perspectives for leaders in knowledge-intensive organizations. Journal of Public Affairs, 18(1). https://doi.org/10.1002/pa.1699

Makani, J., \& Marche, S. (2010). Towards a typology of knowledge-intensive organizations: determinant factors. Knowledge Management Research \& Practice, 8, 265-277.

Makani, J., \& Marche, S. (2012). Classifying organizations by knowledge intensity-necessary next-steps. Journal of Knowledge Management, 16(2), 243-266. 
Mathew, J. (2019). Organisational culture and effectiveness: a multi-perspective evaluation of an Indian knowledge-intensive firm. Employee Relations: The International Journal, 41(3), 538-551. https://doi.org/10.1108/ER-09-2017-0219

Mattarelli, E., Bertolotti, F., \& Incerti, V. (2015). The interplay between organizational polychronicity, multitasking behaviors and organizational identification: a mixed-methods study in knowledge intensive organizations. International Journal of Human Computer Studies, 79, 6-19. https://doi.org/10.1016/j.ijhcs.2015.02.002

Mayan, M. J. (2009). Essentials of qualitative inquiry. California, usa: Left Coast Press.

Méndez, C. E. (2006). Metodología: diseño y desarrollo del proceso de investigación con énfasis en ciencias empresariales. México: Editorial Limusa.

Meredith, J. (1993). Theory building through conceptual methods. International Journal of Operations \& Production Management, 13(5), 3-11.

Miles, I. D., Belousova, V., \& Chichkanov, N. (2018). Knowledge intensive business services: ambiguities and continuities. Foresight, 20(1), 1-26. https://doi.org/10.1108/FS-10-2017-0058

Millar, C. C. J. M., Chen, S., \& Waller, L. (2017). Leadership, knowledge and people in knowledge-intensive organisations: implications for HRM theory and practice. International Journal of Human Resource Management, 28(2), 261-275. https://doi.org/10.1080/09585192.20 16.1244919

Mohammadi, A. (2019). Determining appropriate zones for knowledge intensive firms site selection using GIs: case study of Tehran metropolitan area. International Journal of Applied Geospatial Research, 10(1), 1-30. https://doi.org/10.4018/IJAGR.2019010101

Mol, M. J., \& Brandl, K. (2018). Bridging what we know: the effect of cognitive distance on knowledge-intensive business services produced offshore. International Business Review, 27(3), 669-677. https://doi.org/10.1016/j.ibusrev.2017.11.003

Morten, P., \& Syvertsen, T. G. (1998). Coordination in knowledge-intensive organization. Lecture Notes in Computer Science, 1364, 9-23.

Naisbitt, J. (1983). Macrotendencias: diez nuevas orientaciones que están transformando nuestras vidas. Barcelona: Mitre.

Nicolaescu, S. S., Florea, A., Kifor, C. V., Fiore, U., Cocan, N., ... Zanetti, P. (2020). Human capital evaluation in knowledge-based organizations based on big data analytics. Future Generation Computer Systems, 111, 654-667. https://doi.org/10.1016/j.future.2019.09.048

Nonaka, I., \& Takeuchi, H. (1999). La organización creadora de conocimiento: cómo las compañias japonesas crean la dinámica de la innovación. México: Oxford.

Nurmi, R. (1998). Knowledge-intensive firms. Business Horizons, 41(3), 26-32. 
Penco, L., Ivaldi, E., Bruzzi, C., \& Musso, E. (2020). Knowledge-based urban environments and entrepreneurship: inside eu cities. Cities, 96. https://doi.org/10.1016/j.cities.2019.102443

Robertson, M., \& O'Malley, G. (2000). Knowledge management practices within a knowledge-intensive firm: the significance of the people management dimension. Journal of European Industrial Training, 24(2), 241-253. http://doi.org/10.1108/03090590010321205

Robertson, M., \& Swan, J. (2003). Control - What control? Culture and ambiguity within a knowledge intensive firm. Journal of Management Studies, 40(4), 831-858.

Santos, J. B. (2020). Knowledge-intensive business services and innovation performance in Brazil. Innovation \& Management Review, 17(1), 58-74. https://doi.org/10.1108/INMR-03-2019-0025

Seto, I., Arkison, B., \& Williams, K. (2011). Knowledge management in a small knowledge intensive organisation: research design process at the Cochrane Child Health Field. Knowledge Management Research \& Practice, 9, 378-384. http://doi.org/10.1057/kmrp.2011.31

Shi, X., Wu, Y., \& Zhao, D. (2014). Knowledge intensive business services and their impact on innovation in China. Service Business, 8, 479-498. http://doi.org/10.1007/s11628-0130202-1

Škerlavaj, M., Dimovski, V., \& Desouza, K. C. (2010). Patterns and structures of intra-organizational learning networks within a knowledge-intensive organization. Journal of Information Technology, 1-16.

Sokeila, I., Mäkines, S., \& Näsi, J. (2003). The strategy logic of personnel in a knowledge-intensive organizations. Frontiers of E-Business Research, 393-407.

Song, Y., Zhang, L., \& Shi, Y. (2007). Study on knowledge transfer and sharing of the core process within knowledge-intensive organizations. In $14^{\text {th }}$ International Conference on Management Science \& Engineering, The National Natural Science Foundation of China, Harbin, P. R. China.

Starbuck, W. (1992). Learning by knowledge-intensive firms. Journal of Management Studies, $29(6), 0022-2280$.

Starbuck, W. (1993). Keeping a butterfly and an elephant in a house of cards: the elements of exceptional success. Journal of Management Studies, 30(6), 885-921.

Sveiby, K. (1992). The knowhow company: strategy formulation in knowledge-intensive industries. International Review of Strategic Management, 3, 167-186.

Sveiby, K. (2001). A knowledge-based theory of the firms to guide in strategy formulation. Journal of Intellectual Capital, 2(4), 344-358.

Sveiby, K. E., \& Lloyd, T. (1987). Managing knowhow. London: Bloomsbury. 
Swart, J., \& Kinnie, N. (2003). Sharing knowledge in knowledge-intensive firms. Human Resource Management Journal, 13(2), 344-358.

Sydänmaanlakka, P. (2002). Intelligent organization. Oxford: Capstone.

Tuominen, T., \& Martinsuo, M. (2019). Employees' agency in the formalisation of knowledge-intensive business service processes: a cross-case comparison. Journal of Service Theory and Practice, 29(1), 45-70, https://doi.org/10.1108/JSTP-10-2017-0184

Winch, G., \& Schneider, E. (1993). Managing the knowledge-based organizations: the case of architectural practice. Journal of Management Studies, 30(6), 0022-2380.

Winter, S. (1998). Knowledge and competence as strategic assets. In D. Teece (Ed.), The competitive challenge (pp. 159-184). Cambridge, MA: Ballinger.

Wyrwich, M. (2019). New KIBS on the bloc: the role of local manufacturing for start-up activity in knowledge-intensive business services. Regional Studies, 53(3), 320-329. https://doi. org/10.1080/00343404.2018.14780

Yalabik, Z. Y., Swart, J., Kinnie, N., \& Van Rossenberg, Y. (2017). Multiple foci of commitment and intention to quit in knowledge-intensive organizations (кіоs): what makes professionals leave? International Journal of Human Resource Management, 28(2), 417-447. https://doi.org/10.1080/09585192.2016.1244918

Zhang, C. U. I. (2019). Agglomeration economies and performance in knowledge-intensive business services. Singapore Economic Review. https://doi.org/10.1142/S0217590819500048

Zhou, D., Kautonen, M., Wang, H., \& Wang, L. (2017). How to interact with knowledge-intensive business services: a multiple case study of small and medium manufacturing enterprises in China. Journal of Management and Organization, 23(2), 297-318. https://doi. org/10.1017/jmo.2016.23 